\title{
Adrenergic Regulation of Lipolysis In Situ at Rest and during Exercise
}

Peter Arner, Eva Kriegholm, Peter Engfeldt, and Jan Bolinder

Department of Medicine and Research Center, Huddinge Hospital, S-141 86 Huddinge, Sweden

\begin{abstract}
The adrenergic regulation of lipolysis was investigated in situ at rest and during standardized bicycle exercise in nonobese healthy subjects, using microdialysis of the extracellular space in subcutaneous adipose tissue. The glycerol concentration was about two times greater in adipose tissue than in venous blood. At rest, the glycerol concentration in adipose tissue was rapidly increased by $100 \%(P<0.01)$ after the addition of phentolamine to the ingoing perfusate, whereas addition of propranolol did not alter the adipose tissue glycerol level. Glycerol in adipose tissue and plasma increased during exercise and decreased in the postexercise period. Propranolol in the perfusate almost completely inhibited the increase in the tissue dialysate glycerol during the exercise-postexercise period. Phentolamine, however, was completely ineffective in this respect. During exercise, the lipolytic activity was significantly more marked in abdominal than in gluteal adipose tissue; this was much more apparent in women than in men. Thus, in vivo lipolysis in subcutaneous adipose tissue is regulated by different adrenergic mechanisms at rest and during exercise. Alphaadrenergic inhibitory effects modulate lipolysis at rest, whereas beta-adrenergic stimulatory effects modulate lipolysis during exercise. In addition, regional differences in lipolysis are present in vivo during exercise, which seem governed by factors relating to sex. (J. Clin. Invest. 1990. 85:893-898.) fat cell $\cdot$ glycerol $\cdot$ catecholamines
\end{abstract}

\section{Introduction}

The mobilization of lipids from adipose tissue plays a key role in the regulation of the energy balance at rest and during exercise. Free fatty acids derived from lipolysis in fat cells constitute the major fuel used during prolonged exercise and long term anabolic situations. It is not clear which factors are involved in the regulation of lipolysis at rest and during exercise in vivo $(1,2)$. In man, the sympathetic nervous system probably plays an important role, because catecholamines are the only hormones with a marked lipolytic effect on the fat cells of adult subjects (3). In theory, all adrenoceptor subtypes may be involved in the regulation of lipolysis, since binding sites for

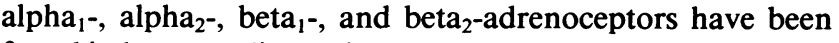
found in human adipose tissue (4). Adrenergic effects on blood flow may also be involved in the regulation of lipolysis (5). In addition, the results of recent in vitro studies (6) suggest that

Address reprint requests to Dr. P. Arner, Department of Medicine, Huddinge Hospital, S-141 86 Huddinge, Sweden.

Received for publication 18 May 1989 and in revised form 23 October 1989.

J. Clin. Invest.

(C) The American Society for Clinical Investigation, Inc.

0021-9738/90/03/0893/06 \$2.00

Volume 85, March 1990, 893-898 human lipolysis is constantly inhibited by the action of hormones and parahormones such as insulin, prostaglandins, adenosine, nicotinic acid, and catecholamines (the latter acting via alpha ${ }_{2}$-adrenoceptors). According to this theory, lipolysis is stimulated by the elimination of endogenous inhibition. Finally, the fat cell lipolysis regulatory system can be acutely modified by exercise (7).

Another important aspect of fat mobilization is that there are marked regional differences in the hormonal regulation of lipolysis in human adipose tissue (8). Catecholamines are much more lipolytic in the abdominal than in the gluteal/femoral fat depots. The latter may be of importance for the development of regional forms of adiposity such as android and gynoid obesity (9). The regional variations in lipolysis have been demonstrated only in vitro. It is not known whether they are present in vivo.

The knowledge of human lipolysis is derived from in vitro studies or from measurements of circulating lipolysis metabolites. Previously there was no method available for the direct investigation of lipolysis in vivo in human adipose tissue. However, we have recently developed a method for in situ investigations of adipose tissue lipolysis, using microdialysis (10). With this technique it is possible to continuously monitor glycerol (lipolysis index) in the extracellular space of adipose tissue and at the same time deliver drugs locally to this space through the microdialysis probe, thereby eliminating the generalized effects of the drug.

In this investigation we have studied the adrenergic regulation of lipolysis in situ in the intact subcutaneous adipose tissue of nonobese male and female subjects. Adipose tissue has been microdialysed at rest or during physical exercise, using dialysis solvents with or without adrenoceptor blocking agents.

\section{Methods}

Subjects. The study group comprised healthy nonobese drug-free volunteers (eight men and nine women) of normal weight. The age (mean \pm SE) was $32 \pm 3 \mathrm{yr}$ in men and $37 \pm 4 \mathrm{yr}$ in women. The body mass index $\left(\mathrm{kg} / \mathrm{m}^{2}\right)$ was $24.2 \pm 0.05$ in men and $21.5 \pm 0.7$ in women. The waist/hip-ratio was $0.953 \pm 0.011$ in men and $0.871 \pm 0.039$ in women $(P<0.01)$. They all undertook regular moderate exercise but no one was involved in athletic performances. The subjects were investigated on one or two occasions. The study was approved by the Ethics Committee of the Karolinska Institute. The subjects were given a detailed description of the study and their consent was obtained.

Microdialysis probe. The microdialysis probe was from Carnegie Medicin, Stockholm, Sweden. It has been described in detail previously (11). Briefly, dialysis tubing ( $10 \times 0.5 \mathrm{~mm}, 20,000 \mathrm{MW}$ cutoff) was glued to the end of a double steel cannula. The perfusion solvent enters the probe through the inner cannula, passes down to the tip of the probe, streams upwards in the space between the inner cannula and the outer dialysis membrane, and leaves the probe through the outer cannula via a side-arm from which it is collected.

Experiment protocol. The experiments performed at rest were conducted as follows. The subjects were investigated in the supine position 
at 8 a.m. after an overnight fast. With a guide cannula (diameter 1.4 $\mathrm{mm}$ ) the dialysis probe was inserted percutaneously without anesthesia into the abdominal subcutaneous adipose tissue in a square $(10 \mathrm{~cm}$ $\times 10 \mathrm{~cm}$ ) immediately to the right of the umbilicus or in the upper lateral part of the gluteal region. Dependent upon the type of experiment, one to three dialysis probes were used in each occasion. The number of probes used in each type of experiment is stated in the legends to the figures. The distance between each probe was always 3 $\mathrm{cm}$. The inlet tubing of the probe was connected to a microinjection pump (Carnegie Medicin) and was continuously perfused $(5 \mu \mathrm{l} / \mathrm{min})$ with Ringer's solution. Adrenergic receptor blocking agents were added as sterile solutions to the ingoing perfusate. This included propranolol (ICI, Chester, England) and phentolamine (Ciba-Geigy, Basel, Switzerland). In each experiment, 15-min fractions of the outgoing dialysate were collected for the analysis of glycerol. The first 15min fraction was deleted, since methodological experiments (12) have shown that there is a transient rise in ATP concentration in the outgoing dialysate during the first $15 \mathrm{~min}$ of dialysis, which is probably due to the initial trauma caused by insertion of the dialysis probe into the adipose tissue. In vitro recovery experiments were performed as described previously (10). At a perfusion velocity of $5 \mu \mathrm{l} / \mathrm{min}$, the in vitro recovery for glycerol is $\sim 17 \%$. During the dialysis experiments, venous blood samples were drawn at 15 -min intervals from an indwelling polyethylene catheter placed in the cubital vein. The first sample was obtained immediately before the probes were inserted in the subcutaneous adipose tissue. Thereafter blood samples were obtained in the middle of each microdialysis fraction.

The exercise experiments were performed at 8 a.m. after an overnight fast with the subjects seated on a bicycle ergometer. The dialysis probes were inserted and dialysis was performed in the exact same manner as described above, except that 5-min fractions were collected for assay of glycerol. The first three fractions were not used. When the subjects had rested for $35 \mathrm{~min}$ on the bicycle ergometer, they exercised for $30 \mathrm{~min}$ at two thirds of their maximum working capacity. Then they rested on the bicycle ergometer for a further $30 \mathrm{~min}$. Maximum working capacity was assessed as described elsewhere (7). The maximum oxygen consumption $(\mathrm{ml} / \mathrm{kg} \times \mathrm{min})$ was $2.8 \pm 0.1$ in the male subjects and $2.0 \pm 0.2$ in the female subjects. Venous blood samples were drawn at regular intervals for the determination of plasma catecholamines (13) and glycerol, using an indwelling catheter, as described above.

In additional experiments the absolute concentration of glycerol in subcutaneous adipose tissue was determined under steady-state conditions using the in vivo calibration technique (14). The experiments were performed as the resting experiments described above, except that the probe was perfused with Ringer's solution followed by differen glycerol solutions $(50,200,400$, and $800 \mu \mathrm{mol} / \mathrm{liter})$. With this method, a linear relationship is established between the perfusate concentration and the increase or decrease in the dialysate glycerol concentration (14). By regression analysis, the glycerol concentration in the perfusion medium, which is in equilibrium with the extracellular tissue concentration, is determined.

Analysis of glycerol. $25 \mu \mathrm{l}$ of plasma or tissue dialysate were used for the analysis of glycerol, using an ultrasensitive kinetic bioluminescence assay of glycerol (15). An automatic luminescence analyzer (LKB Instruments, Inc., Wallac, Helsinki, Finland) was used for the assay.

Statistical analysis. Values are means \pm SE. The coefficient of variation in duplicate determinations was calculated as the standard deviation divided by the mean. The area under the glycerol curve was determined by addition of the trapezoid parts of the area under the curve. The $t$ test or analysis of variance was used for statistical comparison of the values.

\section{Results}

The glycerol levels in the extracellular space were continuously monitored for $2 \mathrm{~h}$ at rest using two different microdialysis

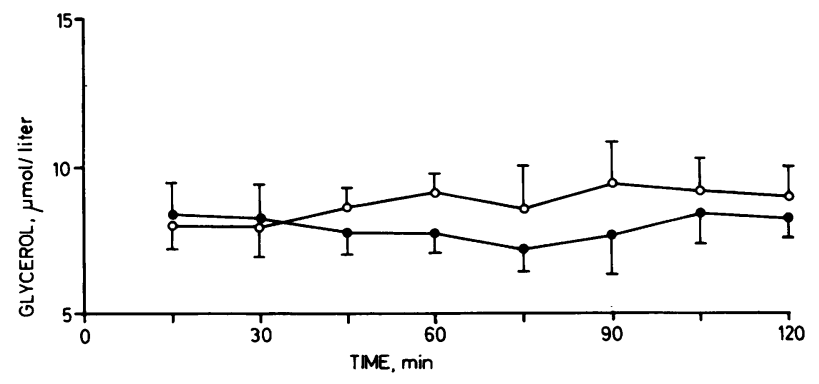

Figure 1. Comparison of glycerol levels at rest in subcutaneous adipose tissue dialysate from two different dialysis probes in the same subjects. The probes were implanted in the abdominal area at a distance of $30 \mathrm{~mm}$ and perfused with Ringer's solution $(5 \mu \mathrm{l} / \mathrm{min})$.

After a 15-min equilibrium period, perfusate was collected at $15-\mathrm{min}$ intervals. Values are mean $\pm \mathrm{SE}$ of four experiments.

probes in the same subject (Fig. 1). Ringer's solution was used as the dialysis solvent. The adipose tissue dialysate glycerol level was constant in both dialysates throughout the experiments. The glycerol values in the two probes did not differ statistically at any observation point. There was also no statistical difference in glycerol values between the two probes when analysis of variation was used as the statistical test. The coefficient of variation for the glycerol values between the two probes was low, i.e., $6 \pm 2 \%$ in four subjects. Steady-state plasma glycerol levels were also recorded throughout these experiments (data not shown).

The effect of alpha- and beta-adrenergic blocking agents on adipose tissue glycerol levels at rest was investigated in the experiments shown in Fig. 2. Addition of the nonselective beta-adrenergic blocking agent propranolol $\left(10^{-4} \mathrm{~mol} / \mathrm{liter}\right)$ to the ingoing perfusate solution did not alter the steady-state adipose tissue perfusate glycerol level during a 75-min observation period. However, addition of the nonselective alphaadrenergic blocking agent phentolamine $\left(10^{-4} \mathrm{~mol} / \mathrm{liter}\right)$ to the dialysis perfusate resulted in a rapid and marked increase in the adipose tissue dialysate glycerol concentration at rest. The glycerol level was more than doubled within $1 \mathrm{~h}$ after the addition of phentolamine $(P<0.01)$. The local exposure of adipose tissue to adrenoceptor blockade did not influence the steady-state plasma glycerol level (data not shown).

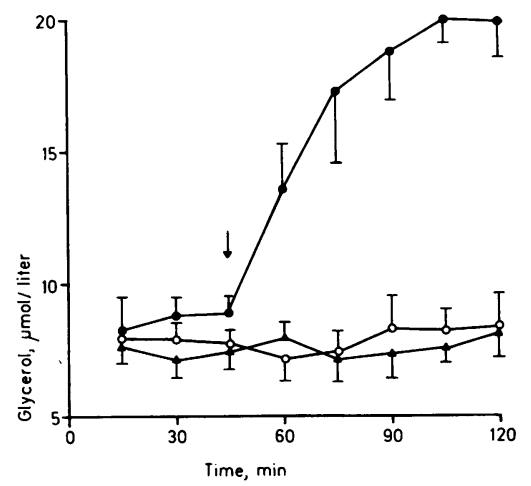

Figure 2. Effect of adrenoceptor blockade on subcutaneous adipose tissue dialysate glycerol at rest. The experiments were conducted as described in Fig. 1. Three different probes were inserted in the same subject in the abdominal area. They were perfused with Ringer's solution alone (open circles) or with either $10^{-4} \mathrm{~mol} / \mathrm{liter}$ of propranolol (triangles) or $10^{-4} \mathrm{~mol} /$ liter of phentolamine ( filled circles). Adrenergic drugs were added after $45 \mathrm{~min}$ of dialysis which is indicated by an arrow. Values are means $\pm \mathrm{SE}$ of four experiments. 


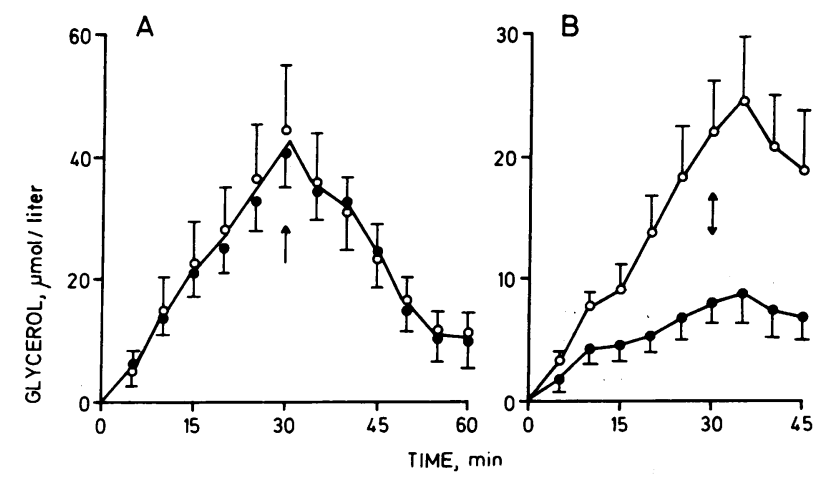

Figure 3. Effect of adrenoceptor blockade on exercise-induced glycerol levels in dialysates of abdominal subcutaneous adipose tissue. Two dialysis probes were inserted in the abdominal area of the same subject and perfused with Ringer's solution either in the absence (open circles) or presence ( filled circles) of either $10^{-4} \mathrm{~mol} / \mathrm{liter}$ of phentolamine $(A)$ or $10^{-4} \mathrm{~mol} /$ liter of propranolol $(B)$. The subjects rested for $35 \mathrm{~min}$. Then they exercised for $30 \mathrm{~min}$ at two thirds of their maximum working capacity. Thereafter, they rested for a further $30 \mathrm{~min}$. After a 15 -min equilibrium period, dialysate was collected at 5-min intervals. The mean glycerol levels in the first four fractions preceeding exercise were determined. This baseline glycerol value was subtracted from the subsequent exercise and postexercise values. Time 0 indicates the start of exercise. The arrow indicates the end of exercise. Values are means \pm SE. $A$ depicts experiments with and without phentolamine performed on two men and six women. $B$ depicts experiments with and without propranolol performed on four men and two women.

It should be noted that the final concentration of propranolol and phentolamine in the extracellular space may be lower than that in the dialysate solvent. The recovery of adrenergic agents under the present conditions is about $10 \%$ (10). However, there is an unknown further dilution of the drugs when they enter the extracellular water space through the dialysis membrane.

Fig. 3 shows the changes in abdominal subcutaneous adipose tissue dialysate glycerol during exercise, when this tissue was microdialyzed with Ringer's solution in the absence or the presence of an adrenergic blocking agent. In the absence of such agents, the concentration of glycerol in the perfusate glycerol gradually increased during the exercise period and peaked at a level which was about 2.5 -fold higher than the baseline level. After exercise, the adipose tissue perfusate glycerol concentration gradually decreased and approached the initial value after $30 \mathrm{~min}$ of rest. The addition of $10^{-4} \mathrm{~mol} / \mathrm{liter}$ of phentolamine to the perfusate did not alter the dialysate glycerol response to exercise. The changes in adipose tissue dialysate glycerol occurring during and after exercise were almost identical with or without the use of phentolamine in the dialysis experiment. When, however, the perfusate was supplemented with $10^{-4} \mathrm{~mol} /$ liter of propranolol, the effect of exercise on adipose tissue dialysate glycerol was markedly reduced. On the average, propranolol inhibited an exercise-induced increase in perfusate glycerol by about two thirds. The effect of propranolol was statistically significant $(P<0.025$ or less comparing individual values; $P<0.01$ using comparison of the area under the curve). The data in Fig. 3 are expressed as changes in glycerol above the baseline value. Similar results were obtained when lipolysis during and after exercise was expressed as a percentage of the baseline glycerol value. In uncharted experiments, it was noted that plasma and adipose tissue dialysate glycerol remained constant for at least $1 \mathrm{~h}$ in the sitting resting position. The results in Fig. 3 represent pooled data from women and men. However, the findings were not altered when we excluded the data obtained from the two men undergoing phentolamine experiments and from the two women undergoing propranolol experiments.

In order to investigate the possible existence of in vivo regional differences in lipolysis, experiments were conducted in which abdominal and gluteal subcutaneous adipose tissues were simultaneously microdialyzed during exercise (Fig. 4). In women, there were marked regional variations in the changes in dialysate glycerol during the exercise and postexercise periods. The gluteal region was much less responsive to exercise than the abdominal region. On the average, the difference was more than threefold and statistically significant $(P<0.05$ or better, comparing individual values; $P<0.01$, comparing area under the curve). When, on the other hand, abdominal and gluteal adipose tissues were simultaneously microdialyzed in men, the regional differences in dialysate glycerol during and after exercise were much less apparent than in women. On the average, abdominal tissue was only about $50 \%$ more responsive to exercise than gluteal tissue in men. This regional difference reached statistical significance only at the end of the exercise period and during the first $15 \mathrm{~min}$ of the postexercise period. The resting adipose tissue dialysate glycerol levels did not differ statistically between the regions or between the sexes.
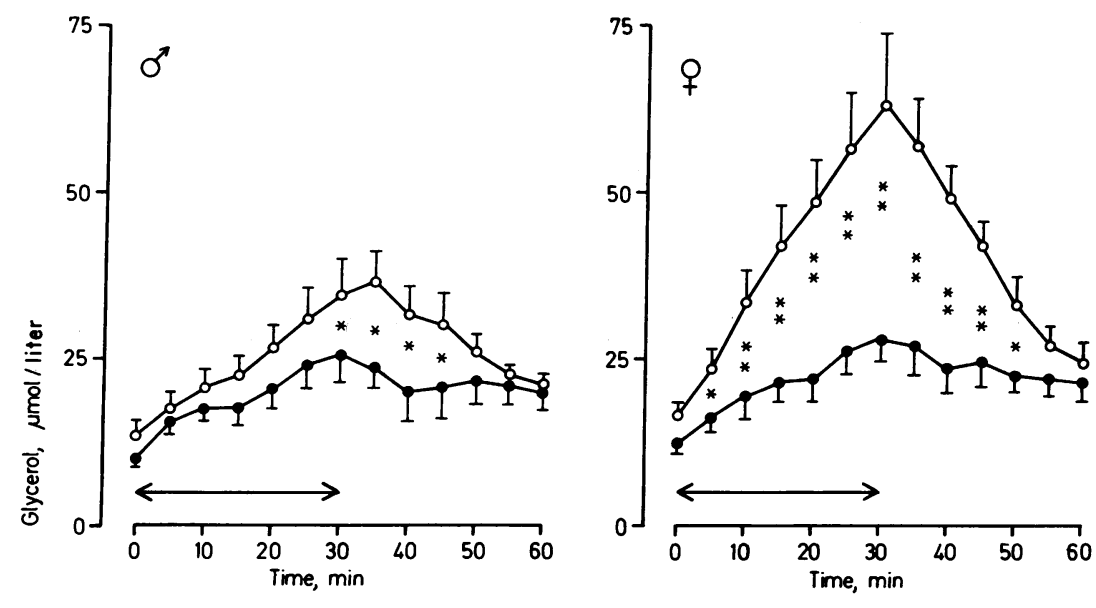

Figure 4. Glycerol levels during exercise in dialysate of abdominal (open circles) and gluteal ( filled circles) subcutaneous adipose tissue of eight men (left graph) and nine women (right graph). The experiments were conducted as described in Fig. 3, using Ringer's solution as the perfusate. Two probes were used simultaneously. One probe was inserted in the abdominal area and one in the gluteal area of each subject. Time 0 represents the baseline glycerol level. The exercise period is indicated by an arrow. The results with abdominal and gluteal adipose tissue were statistically analyzed using $t$ test. Individual values and the area under the curve were compared. Both tests gave essentially the same results. The results of individual comparison are given in the figure. ${ }^{*}, P$ $<0.05$; $^{* *}, P<0.01$. 
Fig. 4 also shows that the change in adipose tissue dialysate glycerol induced by exercise was almost identical in the gluteal region of men and women but was different between the sexes in the abdominal region. The peak in adipose tissue dialysate glycerol level in the abdominal area during exercise was about twice as high in women as in men. This sex difference was statistically significant $(P<0.05$ to $P<0.01$, comparing individual values; $P<0.025$, comparing the area under the curve).

Sex differences may also explain why the adipose tissue glycerol response to exercise was about two times higher in Fig. $3 A$ than in Fig. $3 B$. The former panel denotes experiments with six women and two men. In the latter panel, two women and six men were investigated.

To find out whether alpha-adrenoceptors were involved in the exercise-induced regional differences in adipose tissue dialysate glycerol, experiments were performed in which abdominal and gluteal abdominal tissues were simultaneously dialyzed using Ringer's solution supplemented with $10^{-4} \mathrm{~mol} /$ liter of phentolamine (Fig. 5). In six women, phentolamine did not influence the exercise-induced regional differences in adipose tissue dialysate glycerol. The differences in glycerol,response to exercise between the regions were of the same magnitude and showed the same dynamic changes with phentolamine (Fig. 5) as without phentolamine (Fig. 4).

The in vivo findings in exercising men and women were also compared. Fig. 6 depicts the in vivo results obtained during the dialysis experiments presented in Fig. 4. The resting plasma glycerol level was higher in women than in men $(P$ $<0.05)$. The exercise-induced changes in plasma glycerol were significantly more pronounced in female than in male subjects. However, there were no sex differences as regards pulse or plasma catecholamines before, during, or after exercise (data not shown). It should be noted that the pattern of glycerol changes in plasma and adipose tissue during and after exercise were similar although the absolute values differed.

Although the major reason for the present study was not to investigate the true concentration of glycerol in the extracellular space of adipose tissue, some attempts were made to compare adipose tissue glycerol levels with the plasma glycerol levels at rest. Two women and two men were investigated. Abdominal and gluteal adipose tissues were simultaneously dialyzed and the mean plasma glycerol level during the exper-

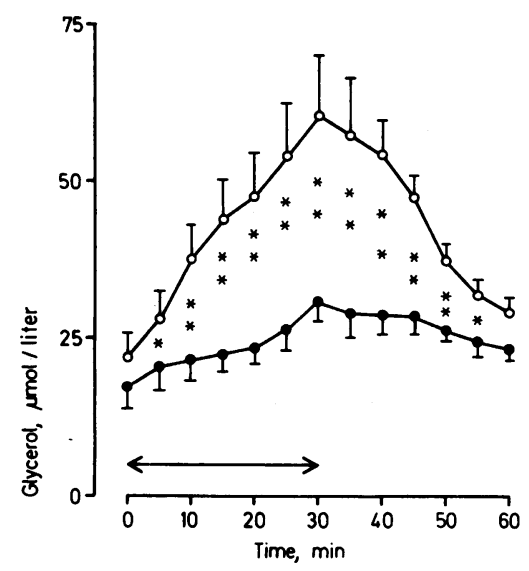

Figure 5. Effect of alpha-adrenoceptor blockade of glycerol levels during exercise in dialysates of abdominal (open circles) or gluteal ( filled circles) subcutaneous adipose tissue of six women. Two probes were used simultaneously in each subject. The experiments were conducted and the results were compared as described in Fig. 4 except that the dialysis solvent (Ringer's solution) was supplemented with $10^{-4} \mathrm{~mol} / \mathrm{liter}$ of phentolamine.

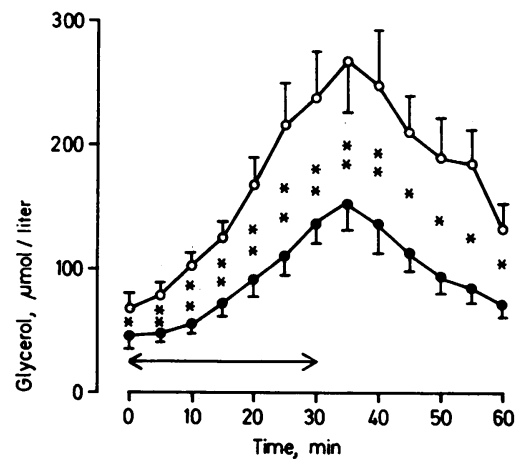

Figure 6. Changes in and plasma levels of glycerol during and after exercise in eight men (filled circles) and nine women (open circles). The results were obtained from the dialysis experiments described in Fig. 4. Time 0 represents the baseline (i.e., resting) value. The results with plasma glycerol were statistically evaluated as described in Fig. 4.

iment was determined. The dialysate solvent was supplemented with increasing concentrations of glycerol and the level of glycerol which is in equilibrium with adipose tissue was determined. The values for glycerol in plasma, abdominal adipose tissue, and gluteal adipose tissue were $61 \pm 6,120 \pm 15$, and $119 \pm 17 \mu \mathrm{mol} /$ liter, respectively. Thus, at rest there was a twofold difference in glycerol concentration between blood and adipose tissue $(P<0.025)$ but no apparent regional variation between the fat depots. For several reasons, however, the equilibrium technique was not employed in experiments depicted in Figs. 1-5. First, it is very time consuming and therefore not suitable for use in connection with other experiments. Secondly, it is necessary to expose adipose tissue to very high concentrations of the investigated metabolite in the equilibrium experiments. Such high glycerol concentrations may artificially alter the following resting or exercise experiments. In the described experiments, the adipose tissue dialysate glycerol level at rest was about $8 \mu \mathrm{mol} / \mathrm{liter}$, using Ringer's solution with no glycerol as perfusate. In vivo glycerol recovery could then be determined by dividing the latter glycerol value with the equilibrium glycerol value. In vivo recovery was found to be about $7 \%$, which is müch lower than in vitro recovery $(17 \%)$.

\section{Discussion}

In this study, the adrenergic regulation of lipolysis in situ has been investigated for the first time in man. This was done by continuously monitoring the glycerol levels in the extracellular space of subcutaneous adipose tissue using a microdialysis technique. This is a new technique for in vivo lipolysis investigations which has recently been developed and evaluated by our laboratory (10). Microdialysis has previously been used mainly to investigate the extracellular space of the rat brain (16).

In the present study, it was found that the exposure of the extracellular space of adipose tissue to a nonselective beta blocking agent did not alter lipolysis at rest (which showed a steady state activity for several hours). This indicates that beta-adrenergic mechanisms are not involved in the regulation of basal (i.e., resting) lipolysis. On the contrary, the local administration of a nonselective alpha-adrenoceptor blocking agent doubled the resting lipolytic activity in subcutaneous adipose tissue. This suggests that resting lipolysis is modulated by alpha-adrenergic inhibition. The findings with propranolol 
in situ are different from previous in vivo results showing a decrease in plasma free fatty acid levels after intravenous propranolol infusions (17). However, the differences may be related to the route of administration of propranolol. When this agent is given intravenously, several neuronal, hormonal, and circulatory factors may be altered which are not influenced by in situ administration of the drug.

The findings with respect to adrenergic regulation during exercise were contrary to those at rest. Hence, local administration of a nonselective beta-adrenoceptor blocking agent markedly inhibited the exercise-induced increase in subcutaneous adipose tissue glycerol. However, the local administration of a selective alpha-adrenoceptor blocking agent did not alter the rise and fall in adipose tissue glycerol during and after exercise. This suggests that beta-adrenergic, but not alphaadrenergic, mechanisms modulate lipolysis during exercise.

Thus, the present results indicate that catecholamines may play different roles in lipid mobilization from subcutaneous adipose tissue at rest as compared with those during physical work. At rest, the inhibitory mechanisms mediated by alphareceptors are of importance, whereas the stimulatory mechanisms mediated by beta-adrenoceptors operate during work. It is not possible at present to say to what extent adipocyte factors or circulatory factors (i.e., blood vessels) contribute to these regulatory mechanisms. Unfortunately, the microdialysis technique cannot distinguish between events occurring at the level of fat cells or at the level of the microcirculation in adipose tissue. Moreover, it is not known which adrenoceptor subtypes are involved in the in vivo regulation of lipolysis at rest and during work. The experimental protocol is elaborate and, for practical reasons, it is not possible at present to simultaneously use agents that selectively block all the four adrenoceptor subtypes in microdialysis experiments.

With this microdialysis technique, it was also possible for the first time to investigate whether regional differences in lipid mobilization occurs in vivo. Exercise was accompanied by a marked rise in adipose tissue glycerol in the abdominal region and a small increase in the gluteal area. This indicates that lipids are mobilized more readily from the former than from the latter adipose region during exercise. Moreover, these regional differences in lipolysis were much more pronounced in women than in men. This suggests that sex hormones or some other regulatory factors related to sex are to some extent responsible for site differences in in vivo lipolysis regulation during exercise. The mechanisms underlying these regional variations are not known at present. However, alpha-adrenergic-mediated effects seem of less importance, since the site differences in the lipolysis of women who exercise were preserved in microdialysis experiments using nonselective alphaadrenoceptor blockade. The findings of regional differences in the lipid mobilization of exercising women may be of interest with regard to female fat distribution. Thus, decreased lipolysis activity during physical work in the gluteal as compared with the abdominal area may to some extent explain why women tend to accumulate subcutaneous fat more readily around the hips than the abdomen.

In an attempt to compare local and circulating glycerol levels, we have also in this study determined the adipose tissue glycerol concentration at rest. The gluteal and abdominal adipose tissue glycerol levels were about two times higher than the plasma glycerol level at rest. Such differences are to be expected, since fat cells are the major source of glycerol. It has recently been shown that the in vivo recovery of glucose by a subcutaneous microdialysis probe is the same in the fasting state and when the circulating glucose level is elevated by glucose infusions (18). Unfortunately, it is not possible to perform such types of experiments with glycerol. However, it is likely that in vivo recovery for glycerol also is the same under basal and stimulated conditions. If so, the maximal glycerol level in adipose tissue should be at least two times higher than that in blood during exercise. It should be noted that we have compared glycerol from adipose tissue with venous samples from a site draining a muscular area (i.e., the cubital vein). However, our venous sample is probably representative for overall changes in plasma glycerol during prolonged experiments, inasmuch as the latter metabolite is not reused by adipose tissue. The close correlation between changes in glycerol occurring in blood and abdominal adipose tissue during exercise/postexercise speaks in favor of this assumption.

It was observed that in vivo recovery for glycerol by the probe was lower than the in vitro recovery. The reason for this discrepancy is unknown at present but could be due to more rapid uptake of glycerol in vitro than in vivo. Similar differences in recovery between in vivo and in vitro conditions have been reported for glucose $(12,14)$.

An additional finding in the present study was that the plasma glycerol level was higher in women than in men at rest and even higher during exercise. The mechanisms responsible for this sex variation are unknown at present, but cannot be described to differences in heart rate or circulating catecholamines. One factor which may be involved is the presently observed higher lipolytic activity in abdominal adipose tissue of women than men during exercise. The plasma glycerol findings are in accord with a recent study (19) in which plasma glycerol levels at rest and during abdominal surgery were higher in women than in men.

For several reasons, it is justified to statistically compare adipose tissue perfusate glycerol levels during exercise in the different regions of men and women, although the absolute concentration of glycerol in the extracellular water was not determined in these experiments, because of methodological reasons discussed previously. First, the resting levels of adipose tissue dialysate glycerol did not differ statistically between the sexes or between the regions. Second, the coefficient of variation for glycerol using two microdialysis probes in the same individual is very low (i.e., $6 \%$ ).

It could be argued that the findings with adipose tissue glycerol may be artificially influenced by disruption of the extracellular space made by the insertion of the dialysis probe. For example, diffusion distances could be altered due to overall expansion of the extracellular space and the dialysis unit could disrupt the surface of tissue cells. However, the results of previous methodological experiments suggest that the dialysis probe cause only little damage to adipose tissue. First, there is only an initial and transient evidence of cell damage (12). Second, identical changes in subcutaneous adipose tissue glycerol were observed in two different probes in the same animal after intravenous injection of isoproterenol to rats (10).

In summary, the present study shows different kinds of adrenergic regulation of lipolysis in situ in the resting and in working man. At rest, subcutaneous adipose tissue lipolysis seems to be modulated by inhibitory mechanisms that are mediated via alpha-adrenoceptors. During physical work, lipolysis in this tissue appears to be modified mainly by stimula- 
tory effects which are mediated by beta-adrenoceptors. In addition, there are marked regional variations in lipolysis during exercise that favor lipid mobilization from the abdominal rather than from the gluteal fat depots. This finding is much more apparent in women than in men and may be an important factor in the development of regional obesity in females. Finally the investigation also suggests that microdialysis is a suitable technique for investigations of in vivo lipolysis regulation in humans.

\section{Acknowledgments}

This study was supported by grants from the Swedish Medical Research Council (19X-01034), the Swedish Diabetes Association, the Swedish Athletics Council, the Karolinska Institute, and the Foundations of Osterman, Nordic Insulin, Fernström, Wiberg, and Old Female Servants.

\section{References}

1. Davies, J. I., and J. E. Souness. 1981. The mechanisms of hormone and drug actions on fatty acid release from adipose tissue. Rev. Pure \& Appl. Pharmacol. Sci. 2:1-112.

2. Bülow, J., and J. Madsen. 1986. Regulation of fatty acid mobilization from adipose tissue during exercise. Scand. J. Sports Sci. 8:19-26.

3. Marcus, C., H. Ehrén, P. Bolme, and P. Arner. 1988. Regulation of lipolysis during the neonatal period: importance of thyrotropin. $J$. Clin. Invest. 82:1793-1797.

4. Fain, J. N., and J. A. Garcia-Sáinz. 1983. Adrenergic regulation of adipocyte metabolism. J. Lipid Res. 24:945-986.

5. Hjelmdahl, P., and B. Linde. 1985. Adrenergic control of blood flow and lipolysis in human adipose tissue. In Alpha-Adrenoceptor Blockers in Cardiovascular Disease. H. Refsum and O. D. Mjos, editors. Churchill Livingstone, Edinburgh. 151-164.

6. Kather, H., W. Bieger, G. Michel, K. Aktories, and K. H. Jakobs. 1985. Human fat cell lipolysis is primarily regulated by inhibitory modulators acting through distinct mechanisms. J. Clin. Invest. 76:1559-1565.
7. Wahrenberg, H., P. Engfeldt, J. Bolinder, and P. Arner. 1987 Acute adaption of lipolysis during physical exercise in humans. Am. J. Physiol. 253:E383-E390.

8. Arner, P. 1989. Regulation of lipolysis in different regions of human adipose tissue. In Obesity in Europe. P. Björntorp, and S. Rössner, editors. John Libbey and Co. Ltd., London. 177-183.

9. Arner, P. 1988. Control of lipolysis and its relevance to development of obesity in man. Diabetes Metab. Rev. 4:507-515.

10. Arner, P., J. Bolinder, A. Eliasson, A. Lundin, and U. Ungerstedt. 1988. Microdialysis of adipose tissue and blood for in vivo lipolysis studies. Am. J. Physiol. 255:E737-E742.

11. Tossman, U., and U. Ungerstedt. 1986. Microdialysis in the study of extracellular levels of amino acids in the rat brain. Acta Physiol. Scand. 128:9-14.

12. Bolinder, J., E. Hagström, U. Ungerstedt, and P. Arner. 1989. Microdialysis of subcutaneous adipose tissue in vivo for continuous glucose monitoring in man. Scand. J. Clin. Lab. Invest. 49:465-474.

13. Hallman, H., L. E. Farnebo, B. Hamberg, and G. Jonsson. 1978. A sensitive method for determination of plasma catecholamines using liquid chromatography with electrochemical detection. Life Sci. 23:1049-1052.

14. Lönnroth, P., P. A. Jansson, and U. Smith. 1987. A microdialysis method allowing characterization of the intercellular water space in humans. Am. J. Physiol. 253:E228-E231.

15. Björkhem, J., P. Arner, A. Thore, and J. Östman. 1981. Sensitive kinetic bioluminescent assay of glycerol release from human fat cells. J. Lipid Res. 22:1142-1147.

16. Ungerstedt, U. 1984. Measurements of neurotransmittor release by intracranial dialysis. In Measurements of Neurotransmittor Release In Vivo. C. A. Marsden, editor. John Wiley \& Sons, New York. 81-105.

17. Burns, T. W., J. M. Mohn, P. E. Langley, R. Yawn, and G. R. Chase. 1974. Regulation of human lipolysis. In vivo observations on the role of adrenergic receptors. J. Clin. Invest. 53:338-341.

18. Jansson, P. A., J. Fowelin, U. Smith, and P. Lönnroth. 1988. Characterization by microdialysis of intercellular glucose levels in subcutaneous tissues in humans. Am. J. Physiol. 255:E218-E220.

19. Håkansson, E., H. Rutberg, L. Jorfeldt, and L. Wiklund. 1984 Endocrine and metabolic responses after standardized moderate surgical trauma: influence of age and sex. Clin. Physiol. (Oxf.). 4:461-473. 